\title{
Individualized Diet and Lifestyle Modifications Reverse Symptoms of Systemic Lupus Erythematosus
}

\author{
Daniel Rothman ${ }^{1}$; Faiz Khan ${ }^{2}$, MD; Vanessa Rudin ${ }^{1}$ \\ ${ }^{1}$ Mymee Inc, New York, NY, United States \\ ${ }^{2}$ CityMD, New York, NY, United States
}

\section{Corresponding Author:}

Daniel Rothman

Mymee Inc

New York, NY,

United States

Email: daniel@mymee.com

\begin{abstract}
Background: There is increasing evidence that digital therapeutic tools can bring personalized medicine to the masses improving outcomes and decreasing cost. Many factors influence the expression of autoimmune disease, and understanding and removing these underlying triggers provides an opportunity to minimize use of medications and some patients can achieve sustained remission if the right triggers for a patient are identified. This randomized controlled trial is the first to test the efficacy of a digital therapeutic intervention which combines adaptive patient generated health data tracking with health coaching to identify factors triggering lupus and to evaluate reductions in symptoms, and improvements in quality of life.
\end{abstract}

Objective: Evaluate impact of personalized dietary and environmental interventions on quality of life and health care costs.

Methods: This a randomized controlled trial using a convenience sample. The Mann-Whitney U test determined that a sample size of 40 patients ( 20 intervention and 20 control) provides a power of $80 \%$ for continuous and ordinal variables. The Bonferroni Correction was used to ensure that the probability of a type 1 error is less than $5 \%$ even with the large number of hypotheses being tested. Subjects in both groups received standard of care from their physicians. All subjects repeated the online questionnaires (Fatigue Scale - FACIT, Lupus QOL, and BPI-SF) at weeks 4, 8, 12, and 16. The experimental group going through the mymee protocol received health coaching (weekly calls to educate and implement changes based on data analysis). Digital data collection and tracking was used to correlate dietary/lifestyle/environmental patterns with symptoms. The control group completed the same assessments during the 16 week intervention period but did not receive any additional coaching. The intervention uses correlations between symptoms and triggers that are reported daily to create an iterative cycle of hypotheses tailored to each individual. With 5 min of tracking a day using the app, patients easily report what they have eaten, other triggers, and their symptoms. Using our machine learning platform, coaches identify each patient's personal disease triggers and help patients implement changes to remove them.

Results: The interim results of the study showed that $78 \%$ improved in the experimental group and $36 \%$ in the control group with a $P<.01$. Furthermore, $67 \%$ of the patients have gone off some or all of their drugs after consulting with their doctors.

Conclusions: Results show a significant improvement for lupus patients who completed the protocol, demonstrating the potential for digital therapeutics to dramatically improve the quality of life for patients diagnosed not only with lupus, but other chronic diseases ( $80 \%$ of the trial patients also had rheumatoid arthritis). Broad adoption of the mymee intervention could assist in building a database of lupus triggers and symptoms that could lead to further understanding the causes of lupus. Based on interim results, a 78/36 effect size is competitive if not more effective than current drugs in the pipeline like Stelara which shows a 60/31 effect size and includes potential side effects of drug-induced MS and cancer.

(iproc 2018;4(2):e11804) doi: $10.2196 / 11804$

\section{KEYWORDS}

population health; digital health; digital therapeutics; lupus; precision medicine; personalized medicine; chronic disease 
Edited by T Hale; this is a non-peer-reviewed article. Submitted 02.08.18; accepted 29.08.18; published 17.09.18.

Please cite as:

Rothman D, Khan F, Rudin V

Individualized Diet and Lifestyle Modifications Reverse Symptoms of Systemic Lupus Erythematosus

iproc 2018;4(2):e11804

URL: http://www.iproc.org/2018/2/e11804/

doi: $\underline{10.2196 / 11804}$

PMID:

(CDaniel Rothman, Faiz Khan, Vanessa Rudin. Originally published in Iproceedings (http://www.iproc.org), 17.09.2018. This is an open-access article distributed under the terms of the Creative Commons Attribution License (https://creativecommons.org/licenses/by/4.0/), which permits unrestricted use, distribution, and reproduction in any medium, provided the original work, first published in Iproceedings, is properly cited. The complete bibliographic information, a link to the original publication on http://www.iproc.org/, as well as this copyright and license information must be included. 